Words total: 7218

Figures: 0

Tables: 4

\title{
Daily Affect Regulation in Major Depression and Borderline Personality Disorder
}

Ulrike Zetsche*1, Paul-Christian Bürkner ${ }^{3}$, Julian Bohländer ${ }^{1}$, Babette Renneberg ${ }^{1}$, Stefan

$$
\text { Roepke }^{4}, \& \text { Lars Schulze }{ }^{1,2}
$$

1 Clinical Psychology and Psychotherapy, Freie Universität Berlin, Berlin, Germany;

2 Outpatient Psychotherapy Treatment Center at Freie Universität Berlin, Germany;

3 Cluster of Excellence SimTech, University of Stuttgart, Stuttgart, Germany;

4 Department of Psychiatry, Charité - Universitätsmedizin Berlin, Campus Benjamin Franklin, Berlin, Germany

*Corresponding author: Ulrike Zetsche, Freie Universität Berlin, Clinical Psychology and Psychotherapy, Habelschwerdter Allee 45, 14195 Berlin, Germany, phone: +49-30-838 55925; fax: +49-30-838 4 55925; u.zetsche@,fu-berlin.de 
RUNNING HEAD: AFFECT REGULATION IN BPD AND MDD

\begin{abstract}
Emotional disturbances are an inherent aspect of most mental disorders and possibly driven by impaired affect regulation. It is unclear, though, how exactly affected individuals differ from healthy individuals in regulating their affect and whether individuals suffering from different mental disorders face similar or distinct difficulty in affect regulation.

The present study addresses these questions while overcoming earlier methodological constraints. It uses a seven-day experience sampling assessing the employment and effectiveness of six regulation strategies real-time in 55 individuals with current major depressive disorder, 52 individuals with borderline personality disorder (BPD), and 55 healthy individuals.
\end{abstract}

Both clinical groups employed rumination and suppression more often and acceptance less often than healthy individuals. Depressed individuals ruminated even more often than individuals with BPD. All participants rarely employed cognitive reappraisal with no differences between groups. Remarkably, if clinical groups selected adaptive strategies, they were as effective as healthy individuals in implementing them.

Keywords: Emotion regulation, ambulatory assessment, borderline personality disorder, major depressive disorder, rumination, suppression, reappraisal, acceptance 
RUNNING HEAD: AFFECT REGULATION IN BPD AND MDD

\section{INTRODUCTION}

Emotional disturbances are an inherent aspect of most mental disorders. Affected individuals often experience intense feelings of dysphoria, anxiety, euphoria, or a mix of these. In major depressive disorder (MDD), aberrant levels of negative affect are even a cardinal diagnostic symptom. Currently depressed individuals typically experience high levels of inert negative affect as well as large but slow shifts in affect (Nelson et al., 2018). Another condition with remarkably disturbed affect is borderline personality disorder (BPD). In contrast to depressed individuals, individuals with BPD experience instable affective states with sudden large changes in affect (e.g., Ebner-Priemer et al., 2007).

One possible cause for such affective disturbances may be difficulties in regulating ones' affect ${ }^{1}$. Indeed, theoretical models of several disorders highlight deficits in the regulation of affective states as a major cause for the respective affective disturbances (e.g., Joormann \& Stanton, 2016; Linehan, 1993). Affect regulation, however, is a broad construct relating to a variety of processes (Koole, 2009). One of the most influential models (Gross, 1998; Sheppes et al., 2015) conceptualizes affect regulation as a set of strategies individuals implement at different stages of the emotion generation process in order to manage their affective experiences. A comprehensive meta-analysis identified three such regulation strategies as being effective in down-regulating negative affect, at least if healthy individuals are instructed to do so in a laboratory context (Webb et al., 2012). These were cognitive reappraisal, i.e., changing one's appraisal of an emotion eliciting situation, acceptance of one's emotional response, and distraction from the emotion eliciting situation or the emotional experience. In contrast, rumination showed clear negative consequences on individuals' affect. Finally, suppression of one's emotional expression showed no effect on individuals' emotional experience and even

\footnotetext{
${ }^{1}$ Experts argue that a clear distinction between emotion-, mood-, or affect-regulation is not possible. All terms denote changes in emotionally charged states regarding valence or arousal (Koole, 2009). We will use the term affect regulation.
} 
RUNNING HEAD: AFFECT REGULATION IN BPD AND MDD

negative effects on individuals' physiological responding. Following this model, deficits in affect regulation may take many different forms. Individuals with mental disorders may underuse effective regulation strategies, overuse maladaptive regulation strategies, or both. They may also attempt to employ effective strategies but fail to implement them successfully.

The past decades have seen an increasing amount of studies examining differences in affect regulation between healthy individuals and individuals with mental disorders. However, with very few excecptions, studies on the employment of affect regulation strategies used retrospective self-report measures asking individuals what they usually did to regulate their affect. Individuals with mental disorders, though, have been demonstrated to exhibit systematic biases in the recollection of affective states and emotional events (e.g., Ben-Zeev \& Young, 2010; Ebner-Priemer et al., 2006; Zetsche et al., 2019). Thus, results based on restrospective global self-reports have to be interpreted with caution (see also, Lincoln et al., 2022). Studies into the effectiveness of certain regulation strategies mostly used experimental designs examining the effect of instructed strategy use on experimentally induced affect. Experimental studies have high internal validity but are limited in their ecoglogical validity: affective states induced by looking at pictures or videoclips may be less intense and less self-relevant than affective states experienced by personal events. Furthermore, detailed instructions on when and how to implement certain affect regulation strategies may differ from the spontaneous selection and use of strategies in daily life. Finally, previous research in this field has mostly examined non-clinical samples or has compared one clinical sample to healthy individuals. This makes it difficult to find out whether individuals with different mental disorders face similar or distinct patterns of impaired affect regulation.

The present study was designed to overcome the methodological contraints summarized above by using experience sampling methodology assessing six affect regulation strategies in two clinical samples, i.e., individuals with either current MDD or BPD, and healthy individuals. Below, we will summarize previous findings from self-report or experimental studies 
RUNNING HEAD: AFFECT REGULATION IN BPD AND MDD

comparing the use or effect of these regulation strategies between individuals with current MDD or BPD, and healthy individuals. We will also summarize the few experience sampling studies including diagnosed MDD or BPD groups.

\section{Previous findings comparing affect regulation in MDD to healthy individuals}

Visted and colleagues (2018) conducted a comprehensive meta-analysis on 69 trials using retrospective self-report questionnaires to compare depressed and healthy individuals' habitual use of different affect regulation strategies. The authors found that currently depressed individuals reported to employ maladaptive regulation strategies, i.e., rumination and suppression, considerably more often and adaptive strategies, i.e., cognitive reappraisal and acceptance, less often than healthy controls. There is one experience sampling study confirming that individuals with a current MDD ruminated more often in their daily lives than healthy individuals (Kircanski et al., 2015).

There are a couple of experimental studies investigating whether currently depressed individuals are effective in implementing adaptive affect regulation strategies. Results indicate that individuals with a current MDD are as effective as healthy individuals in using reappraisal, distraction, or acceptance to ameliorate their mood, at least when instructed to do so in a laboratory context (for reviews, see Liu \& Thompson, 2017; Nolen-Hoeksema et al., 2008). The effect of instructed rumination on subsequent affect has been shown to be even more detrimental in currently depressed than in healthy non-dysphoric individuals (Liu \& Thompson, 2017). Similarly, spontaneous rumination in response to naturally occurring stressful events as assessed by a 7-day experience sampling showed more deleterious effects on subsequent mood in currently depressed as compared to healthy individuals (Ruscio et al., 2015).

\section{Previous findings comparing affect regulation in BPD to healthy individuals}

Studies comparing the habitual use of affect regulation strategies in individuals with BPD versus healthy individuals demonstrated that individuals with BPD retrospectively report using expressive suppression and rumination more often, and cognitive reappraisal and 
RUNNING HEAD: AFFECT REGULATION IN BPD AND MDD

acceptance less often than healthy individuals (e.g., Beblo et al., 2010, 2013; Daros \& Williams, 2019; Steenkamp et al., 2015; Zaki et al., 2013).

There is evidence from experimental studies suggesting that individuals with BPD are as effective in implementing cognitive reappraisal instructions as healthy individuals (e.g., Krause-Utz et al., 2019; Lang et al., 2012). Similar to depressed individuals, the detrimental effect of (instructed) rumination on affect seems to be more pronounced in individuals with BPD than in healthy individuals (Selby et al., 2009). Surprisingly, some findings indicate that suppression may have an adaptive function in individuals with BPD. For example, instructed suppression of emotional expressions in response to a sad film clip did not increase urges for self-injurious behavior in individuals with BPD, whereas instructed acceptance did (Svaldi, Dorn, et al., 2012). In addition, instructed suppression of emotional experiences reduced negative emotions and urges for self-injurious behavior in the daily lives of individuals with BPD, as assessed by experience sampling (Chapman et al., 2016).

\section{Previous findings comparing affect regulation between BPD and MDD}

There are only a handful of studies directly comparing affect regulation in currently depressed individuals and individuals with BPD. Results of these studies implicate that both groups habitually use cognitive reappraisal, acceptance, and suppression to similar extends, with mixed findings for rumination (Abela et al., 2003; Fernando et al., 2014; Sauer et al., 2016; Svaldi, Griepenstroh, et al., 2012). However, all of these studies employed retrospective selfreport questionnaires and did not clearly separate the two clinical groups, i.e., a considerable part of individuals with BPD suffered from a current comorbid major depressive episode.

There has only been one previous study comparing the effect of affect regulation strategies on affect in individuals with MDD and BPD (Chapman et al., 2016). The authors examined the effect of instructed emotion suppression or acceptance on daily negative affect using experience sampling methodology. Results showed that individuals with BPD, but not depressed indivduals, experienced a decrease in negative affect in response to instructed 
RUNNING HEAD: AFFECT REGULATION IN BPD AND MDD

emotion suppression. In contrast, only depressed individuals, but not individuals with BPD, experienced a decrease in negative affect in response to instructed acceptance. These results implicate that the effectiveness of different regulation strategies may differ depending on the clinical diagnosis. However, more than a third of participants with BPD suffered from a current comorbid MDD, restricting the interpretation of results.

\section{The present study}

The present study was designed to advance the field by using experience sampling methodology to assess the use of various affect regulation strategies and their effect on subsequent affect realtime in the daily lives of participants. In addition, we compared affect regulation between healthy individuals and two clinical samples, namely individuals with a current MDD and individuals with BPD. We chose these two disorders because affective disturbances are a core symptom in both disorders. Yet, the nature of affective disturbances differs between the two groups, as outlined above. Importantly, we excluded patients with comorbid current MDD and BPD diagnoses to circumvent constraints of previous studies. We assessed six different affect regulation strategies to understand the overall pattern of affect regulation. These were two putatively maladaptive regulation strategies, i.e., rumination and suppression, and four putatively adaptive strategies, i.e., cognitive reappraisal, acceptance, distraction, and social sharing. Affect regulation strategies and affect were assessed eight times a day over seven days.

\section{Hypotheses}

Firstly, we expected that, generally, both clinical groups regulate their affect more often than healthy individuals because they experience negative affect more often (Dixon-Gordon et al., 2015; Lincoln et al., 2022).

Based on previous findings on the habitual use of affect regulation strategies, we expected that both clinical groups employ maladaptive strategies, i.e., rumination and suppression, more often in their daily lives than healthy individuals. Conversely, we expected 
that both clinical groups employ adaptive strategies, i.e. cognitive reappraisal and acceptance, relatively less often than healthy controls. We further expected that individuals within each clinical group select putatively maladaptive strategies, i.e., rumination and suppression, more often than they select putatively adaptive strategies, i.e., cognitive reappraisal and acceptance. Conversely, we expected that healthy individuals select rumination and suppression less often than reappraisal and acceptance. Note, that we did not formulate hypotheses regarding the putatively adaptive strategies distraction and social sharing because previous findings regarding these strategies are yet sparse.

With regard to the effect of regulation strategies on affect, we predicted that the negative effect of rumination on affect is more pronounced in both, individuals with BPD or MDD than in healthy individuals. Further, we predicted that suppression is effective in reducing negative affect only in individuals with BPD. 
RUNNING HEAD: AFFECT REGULATION IN BPD AND MDD

\section{TRANSPARENCY AND OPENNESS}

\section{Preregistration}

The study is part of a preregistered research project (Schulze et al., 2018). The online supplement provides a table listing all minor differences between the study protocol and this manuscript.

\section{Data, materials, code, and online resources}

De-individualized data, all statistical code and (additional) results are available at Open Science Framework (OSF): https://osf.io/m72x8/. All questions asked within the sevenday experience sampling can be found in the methods section. In addition, supplemental material including inclusion and exclusion criteria, clinical measures, demographic and clinical characteristics, and minor differences between the study protocol and this manuscript can be found at the journal's webpage.

\section{Reporting}

We report how we determined our sample size, all data exclusions, all manipulations, and all measures in the study.

\section{Ethical approval}

The study was performed in accordance with the World Medical Association Declaration of Helsinki. The ethics committee at Freie Universität Berlin approved the study protocol (no. 67/2013; Amendment: 136/2017). 


\section{METHODS}

\section{Participants}

All participants were recruited through advertisements in online newspapers and at various sites in Berlin, or through specialized treatment facilities. Interested individuals were first screened by telephone. Final eligibility was then established by face-to-face interviews using the German version of the Structured Clinical Interview for DSM-IV Axis I (SCID-I; Wittchen, Wunderlich, Gruschwitz, \& Zaudig, 1997) and Axis II disorders (SCID-II; Fydrich, Renneberg, Schmitz, \& Wittchen, 1997). The SCID has demonstrated good reliability for the majority of the disorders (Lobbestael et al., 2011; Skre et al., 1991). All interviewers were trained in applicating the SCID and were closely supervised for all interviews.

General and group specific inclusion and exclusion criteria are detailed in the online supplements. The final sample included 52 individuals with BPD, 55 individuals with current MDD, and 55 healthy controls (HC). The sample size slightly exceeds our a priori power analysis suggesting a sample size of $\mathrm{N}=159(\mathrm{~N}=53$ per group; for details, see Schulze et al., 2018). All participants identified themselves as female and participants in the three groups were matched for age. Table S1 in the online supplements displays demographic and clinical characteristics by group. Note, that we did not assess ethnic identification, culture background or a measure of income.

All participants gave written informed consent prior to participation and were reimbursed after participating. The study took place in Berlin, Germany, between January 2017 and June 2019.

\section{Materials}

Experience sampling of affect and its regulation in daily life. All participants received a smartphone with a pre-installed experience sampling application (movisensXS; movisens $\mathrm{GmbH}$, Karlsruhe, Germany). The application was programmed to beep eight times 
RUNNING HEAD: AFFECT REGULATION IN BPD AND MDD

a day for seven consecutive days (56 beeps total). The daily sampling period comprised 12 hours divided into eight equal time blocks. The auditory signal occurred pseudo-randomly within each time block. The minimum delay between beeps was 45 minutes. Participants were allowed to postpone prompts three times by $15 \mathrm{~min}$. Responses were time-stamped by the software.

Following each beep, participants were asked to indicate on a scale from 0 (not at all) to 6 (very much) how angry, anxious, ashamed, cheerful, depressed, happy, lonely, and tense they feel ('How did you feel just before the beep?'). Furthermore, participants were asked to indicate on a scale from 0 (never) to 6 (very often) how often they had used several affect regulation strategies since the last beep: rumination ('I thought over and over again about a situation or my feelings'), reappraisal ('I have thought about the situation in a different way'), suppression ('I controlled my emotions by not showing them'), acceptance ('I accepted the situation and/or my feelings), distraction (' $\mathrm{I}$ found an activity to keep myself busy and distracted'), and social sharing ('I found someone to talk about my feelings'; Items based on Heiy \& Cheavens, 2014). Participants received an extra incentive for responding to more than $90 \%$ of beeps.

Overall, compliance with this protocol was very good. The median response rate was $91.07 \%(\mathrm{IQR}=10.48)$ in the BPD group, $92.86 \%(\mathrm{IQR}=8.93)$ in the MDD group, and $89.36 \%$ $(\mathrm{IQR}=15.76 \%)$ in the HC group.

\section{Data analysis}

\section{Statistical Packages and general settings for the models}

We estimated Bayesian hierarchical models to examine our hypotheses. Thus, we applied the R package (R Core Team, 2020) brms (Bürkner, 2017, 2018), which is based on Stan (Carpenter et al., 2017). We always used the default priors of brms, which are chosen to be non- or weakly informative, thus having negligible influence on the obtained results 
RUNNING HEAD: AFFECT REGULATION IN BPD AND MDD

(Bürkner, 2017, 2018). All models reflected the three-level structure of our data (prompts nested in days nested in persons), with varying intercepts over persons. All models included an autoregressive term of order one to reflect the serial correlation of successive prompts within each day. Further, we modelled the within-group variance to vary across groups in all analyses including diagnostic group. For each model, we ran 4 MCMC chains, each with 5000 iterations and 1000 warmup and 4000 post-warmup samples, resulting in a total of 16000 post-warmup samples. All computed models converged with Rhat $=1$ (Vehtari et al., 2021) and estimated effective sample sizes of at least 1200 (or > 540 for the model comparing strategy use within diagnostic groups). There were no divergent transitions. All independent variables are unstandardized. For more information on the convergence of each model and the exact model specifications, see the respective $h t m l$ file at https://osf.io/m $72 \times 8 /$. In the following, we describe the different models used for testing our hypotheses in more detail.

\section{Description of specific models for testing our hypotheses}

To examine whether groups differ in their overall employment of AR strategies, we computed a hierarchical linear model with the mean intensity rating across all AR strategies at time $t$ as dependent variable and group as independent variable.

Next, we examined between group differences in the frequency of employing a particular AR strategy by computing a separate hierarchical linear model for each AR strategy. The dependent variable of each model was the intensity rating for the respective AR strategy at time $t$. Independent variables were group and the mean intensity rating across all other strategies at time $t$ to control for overall strategy use $^{2}$. In addition, we allowed the slope for the mean intensity rating across all other strategies to vary between individuals.

\footnotetext{
${ }^{2}$ Additional analyses on group differences in specific affect regulation strategies are available at OSF (https://osf.io/m72x8/), including uncontrolled group comparisons and group comparisons controlled for mean intensity of all affect regulation strategies, including the strategy at interest.
} 
RUNNING HEAD: AFFECT REGULATION IN BPD AND MDD

To examine whether individuals within each group prefer certain strategies over others, we computed a hierarchical linear model with the intensity rating of any strategy as dependent variable, and the interaction between group and type of strategy as independent variables. We allowed the slope for the type of strategy to vary between individuals and assessment days.

Next, we examined the effect of specific AR strategies on individuals' affect. We first computed an affective valence score by subtracting the mean across all negative affect items from the mean across all positive affect items. Values can range from -6 to 6 , with negative values reflecting negative affective valence, and positive values reflecting positive affective valence. Given floor effects in negative affective states in our healthy individuals, we preferred this valence score over using negative and positive affect scores separately (Ebner-Priemer et al., 2007; Santangelo et al., 2014) ${ }^{3}$. The internal consistency of the affective valence scale was excellent, ESM omega between-person $=0.93(95 \% C I=[0.91,0.95])$. To examine the effect of using a specific AR strategy on the change in affective valence from time $t-1$ to time $t$, we computed hierarchical linear models with the valence score at time $t$ as dependent variable. The lagged person-centered valence score at time $t-1$ and the intensity rating of each AR strategy were entered as independent variables. Due to varying time intervals between experience sampling prompts, we also included the time difference between prompts as independent variable. The time difference between prompts and the lagged valence score were entered within a spline function to model potential non-linear associations with the dependent variable. We further allowed the slope for the intensity rating of each AR strategy and the lagged valence score to vary between individuals.

Finally, we included group as an independent variable into the above model to examine whether the effect of a specific AR strategy on individuals' affect differs between groups.

\footnotetext{
${ }^{3}$ Interested readers can find separate models on negative and positive affect, as outlined in the study protocol, at OSF (https://osf.io/m72x8/).
} 
RUNNING HEAD: AFFECT REGULATION IN BPD AND MDD

We consider effects clearly different from zero if the estimate's 95\% credible interval (i.e., Bayesian confidence interval based on posterior quantiles) does not include zero. When testing directed hypotheses, we estimated the posterior probability $(P P)$ that the respective effect is in the expected direction. $P P$ values range from $0-1$ with higher values implying more support for the effect going into the expected direction. 


\section{Results}

\section{Overall employment of affect regulation strategies}

Table 1a displays mean overall strategy employment by group. As expected, individuals with BPD or MDD generally employed affect regulation strategies clearly more often than healthy individuals (see Table $1 \mathrm{~b}$ for statistics). The two clinical groups did not differ from each other in overall strategy employment.

-Insert Table la about here -

\section{Employment of specific affect regulation strategies}

\section{Between group analyses}

First, we examined whether diagnostic groups differed in their employment of each assessed affect regulation strategy. Given group differences in overall strategy use, we controlled for overall strategy employment by including the mean intensity score across all other strategies into each analysis. Thus, results reflect group differences in the relative employment of each strategy (for results on absolute numbers, see online material at OSF). Table 1a displays the estimated relative employment of each affect regulation strategy by group. Table $1 \mathrm{~b}$ displays the statistics for all between-group contrasts described in the following.

-Insert Table $1 a$ and $1 b$ here -

Maladaptive Strategies. As expected, individuals with BPD or MDD used rumination clearly more often than healthy individuals. Interestingly, depressed individuals used rumination even more often than individuals with BPD. In line with hypotheses, individuals with BPD or MDD also suppressed their emotional expression clearly more often than healthy individuals. However, clinical groups did not differ in how often they used expressive suppression. 
RUNNING HEAD: AFFECT REGULATION IN BPD AND MDD

Adaptive Strategies. Surprisingly, there were no differences between any of the groups in using cognitive reappraisal. As expected, individuals with BPD or MDD accepted their feelings or an external situation clearly less often than healthy individuals. Clinical groups did not differ clearly in how often they used acceptance. Individuals with BPD or MDD distracted themselves clearly more often than healthy individuals. Again, clinical groups did not differ in how often they used distraction. Finally, individuals with BPD or MDD shared their experiences clearly less often with others than healthy individuals. Again, clinical groups did not differ in how often they used social sharing.

\section{Within group analyses}

Next, we examined whether individuals within each group preferred certain strategies over others. As expected, both, individuals with BPD or MDD, selected rumination clearly more often than reappraisal, BPD: $b=0.87(95 \% C I=[0.55,1.20], P P>0.99)$, MDD: $b=1.62$ (95\% $C I=[1.31,1.93], P P>0.99)$. Similarly, individuals with BPD or MDD also selected suppression clearly more often than reappraisal, BPD: $b=1.13(95 \% C I=[0.80,1.48], P P>$ 0.99), MDD: $b=1.16(95 \% C I=[0.82,1.50], P P>0.99)$. Interestingly, only depressed individuals selected acceptance clearly less often than they selected rumination, $b=0.88(95 \%$ $C I=[0.48,1.27], P P>0.99$, or suppression, $b=0.42(95 \% C I=[0.02,0.82], P P=0.98)$. Individuals with BPD selected acceptance to similar degrees than they selected rumination, $b=$ $0.02(95 \% C I=[-0.39,0.43], P P=0.53$, or suppression, $b=0.29(95 \% C I=[-0.12,0.70], P P$ $=0.92$ ). Unexpectedly, healthy individuals did not select reappraisal more often than rumination, $b=-0.15(95 \% C I=[-0.47,0.16], P P=0.83)$, or suppression, $b=-0.15(95 \% C I=$ $[-0.48,0.18], P P=0.82)$. However, they used acceptance clearly more often than they used rumination, $b=-1.29(95 \% C I=[-1.71,-0.88], P P>0.99)$, or suppression, $b=-1.29(95 \% C I$ $=[-1.70,-0.90], P P>0.99)$. 
RUNNING HEAD: AFFECT REGULATION IN BPD AND MDD

\section{Effect of each regulation strategy on subsequent affect}

Table 2a presents the estimated mean effect of each of the six assessed regulation strategies on subsequent affect in the total sample and by group. Table $2 b$ displays the statistics for all between-group contrasts described below.

\section{-Insert Table $2 a$ and $2 b$ here-}

\section{Effect of maladaptive strategies}

In general, participants who ruminated between two experience sampling prompts experienced a clear deterioration of their affect from the previous to the present prompt. When entering the interaction between group and rumination into the model, results showed that, as expected, this negative effect of rumination on affect was clearly more pronounced in individuals with BPD or MDD as compared to healthy individuals. The negative effect of rumination on affect did not differ between clinical groups.

Overall, individuals who suppressed their emotional expressions between two sampling prompts experienced a deterioration of their affect from the previous to the present prompt. When entering the interaction between group and suppression into the model, results showed that this negative effect of suppression was less pronounced in depressed individuals than in healthy individuals. Contrary to our hypothesis, individuals with BPD did not show a positive effect of suppression on affect. Rather, the BPD group did not differ from the other groups in their negative effect of suppression on affect.

\section{Effect of adaptive strategies}

Individuals who used cognitive reappraisal between two sampling prompts experienced a small amelioration of their affect from the previous to the present prompt. When entering the interaction between group and reappraisal into the model, results showed that this small effect of reappraisal on affect did not differ between groups. 
RUNNING HEAD: AFFECT REGULATION IN BPD AND MDD

Overall, individuals who reported to have accepted a situation or their feelings between two experience sampling prompts experienced a clear amelioration of their affect from the previous to the present prompt. When entering the interaction between group and acceptance into the model, results showed that the positive effect of acceptance on affect was clearly more pronounced in individuals with BPD or MDD than in healthy individuals. The positive effect of acceptance on affect did not differ between clinical groups.

Across the entire sample, participants who reported to have distracted themselves from their emotions between two sampling prompts experienced no change in their affect from the previous to the present prompt. Interestingly, when entering the interaction between group and distraction into the model, results showed that healthy individuals distracting themselves experienced a negative effect on their subsequent affect, whereas depressed individuals distracting themselves experienced a positive effect on their subsequent affect. Individuals with BPD experienced no clear effect of distraction on their affect. Accordingly, there were clear differences in the effect of distraction between the MDD and the control group, and the BPD and the control group. There was no clear difference between the MDD and the BPD group.

Individuals who shared their experiences with others between two sampling prompts experienced an amelioration of their affect from the previous to the present prompt. Entering the interaction between group and social sharing into the model did not reveal any group difference in this effect. 
RUNNING HEAD: AFFECT REGULATION IN BPD AND MDD

\section{Discussion}

The present results demonstrate that both, currently depressed individuals and individuals with BPD show an unfavorable pattern of selecting maladaptive affect regulation strategies in their daily lives. Specifically, both clinical groups selected rumination and suppression considerably more often than healthy individuals. Furthermore, they selected effective regulation strategies, such as acceptance and social sharing, relatively less often than healthy individuals. Within-group comparisons roughly reflected this pattern: Both clinical groups selected rumination and suppression more often than they selected cognitive reappraisal. Depressed individuals further selected rumination and suppression more often than acceptance. Conversely, healthy individuals selected acceptance more often than they selected rumination and suppression. This pattern of dysfunctional strategy selection in depressed individuals and individuals with BPD mirrors findings of retrospective questionnaire studies comparing either clinical group to healthy individuals (Daros \& Williams, 2019; Visted et al., 2018).

There are three findings regarding the pattern of strategy selection that are worth to be highlighted: Firstly, the pattern of dysfunctional strategy selection was almost identical in currently depressed individuals and individuals with BPD. The only difference between clinical groups was found for rumination. Depressed individuals ruminated even more often than individuals with BPD. Although individuals with comorbid BPD and MDD have been found to select rumination more often than individuals with MDD or BPD only (Abela et al., 2003, 2003; Dell'Osso et al., 2019), the present results suggest that the strongest association is between rumination and depression. This is in accordance with an extensive body of research identifying rumination as a key mechanism in the development of major depression (Nolen-Hoeksema, 2000).

Second, both clinical groups employed reappraisal relatively as often as healthy individuals. Previous studies comparing either clinical group to healthy individuals have 
RUNNING HEAD: AFFECT REGULATION IN BPD AND MDD

consistently shown that individuals with MDD or BPD report less habitual use of reappraisal than healthy individuals (for reviews, see Daros \& Williams, 2019; Visted et al., 2018). In the present study, however, reappraisal was one of the regulation strategies employed the least often in all groups. Thus, the missing between-group difference in reappraisal use seems to be driven by the low rate of using reappraisal in healthy individuals. This may be a mere sample effect. Else, healthy individuals may only report higher rates of reappraisal when retrospectively asked about their habitual use, but may not actually employ reappraisal that often in their daily lives (see also Heiy \& Cheavens, 2014). Further experience sampling studies are needed to clarify this question.

Third, both clinical groups used distraction extensively and considerably more often than healthy individuals. Similar results were found in another experience sampling study where increased depressive symptoms were associated with increased use of distraction in a non-clinical sample (Panaite et al., 2018). This is in contrast to findings from questionnaire or experimental studies showing that depressed individuals select distraction either less often or to similar degrees than healthy individuals (Liu \& Thompson, 2017). The present results suggest that individuals with MDD or BPD use distraction more often than they remember to do so when asked retrospectively. Interestingly, our result also demonstrated that distraction is effective in improving depressed individuals' affect, but has no effect in individuals with BDP.

Regarding the effectiveness of affect regulation, our results suggest that currently depressed individuals and individuals with BPD were able to benefit from adaptive regulation strategies to similar degrees as healthy individuals if they did select them. Both clinical groups experienced a small positive effect of using reappraisal and a clear positive effect of using acceptance or social sharing. Depressed individuals also benefitted from distraction. These positive effects on affect were either comparable to (i.e., reappraisal, social sharing) or even greater (i.e., acceptance) than in healthy individuals. Remarkably, most strategies showing a 
RUNNING HEAD: AFFECT REGULATION IN BPD AND MDD

positive effect on affect in this study were those employed relatively less often by individuals with MDD or BPD. Thus, despite their intact ability to effectively implement adaptive regulation strategies, both clinical groups do not primarily select them.

We did not replicate findings indicating a positive effect of suppression in individuals with BPD. In our study, individuals with BPD who suppressed their emotional expression experienced a negative effect on their subsequent affect. However, the only previous study demonstrating a positive effect of instructed suppression on affect in clinical BPD instructed patients to suppress their emotional experience rather than their emotional expression (Chapman et al., 2016). Note, that we assessed expressive suppression. Past findings on the effect of expressive suppression in BPD have been mixed. Svaldi and colleagues (2012) found no strategy-specific effect of expressive suppression on self-injurious behavior or negative affect in BPD. In another study, instructions to suppress the experience and expression of emotions when watching a distressing film showed a positive effect on affect independent of BPD symptom severity (Evans et al., 2013). It will thus be important for future research to more clearly differentiate between the suppression of emotional experiences and emotional expressions.

The present results finally demonstrated that the detrimental effect of rumination on affect was more pronounced in both clinical groups as compared to healthy individuals. This is in line with our expectations and also in accordance with findings suggesting that rumination only exerts negative consequences in individuals who are already in a negative mood (NolenHoeksema et al., 2008), as can be assumed for individuals with MDD or BPD.

In sum, the present study is the first to directly compare the employment and effectiveness of six affect regulation strategies in the daily lives of healthy individuals and two carefully diagnosed clinical samples, namely individuals with MDD or BPD. Results demonstrated that both clinical groups face similar problems in affect regulation. Specifically, 
they both showed a marked pattern of maladaptive strategy selection but no deficits in effectively implementing adaptive strategies. It remains an exciting question why individuals with MDD or BPD show a clear preference for affect regulation strategies that actually increase their negative affect. Possibly, they are not able to implement helpful strategies in moments of intense negative affect or high arousal. Alternatively, they may hold erroneous beliefs about a positive effect of these strategies. It will be intriguing for future research to examine this question. Finally, the present results support the notion that difficulty in affect regulation is a transdiagnostic process underlying different kinds of psychopathology (Sloan et al., 2017). This supports the call for transdiagnostic approaches targeting aberrant psychological processes shared by various mental disorders. 
RUNNING HEAD: AFFECT REGULATION IN BPD AND MDD

\section{Author contributions}

Conceptualization: L. Schulze, U. Zetsche, and B. Renneberg; Methodology: C.-P. Bürkner, L. Schulze, and U. Zetsche; Formal Analysis: L. Schulze and U. Zetsche; Investigation: J. Bohländer, and S. Roepke

Writing - Original Draft Preparation: U. Zetsche; Writing - Review \& Editing: P.-C. Bürkner, J. Bohländer, B. Renneberg, S. Roepke, and L. Schulze

\section{Conflicts of interest}

The authors declare that there were no conflicts of interest with respect to the authorship or the publication of this article.

\section{Funding}

This study was funded by two grants from the German Research Foundation (DFG) to Lars Schulze (DFG-SCHU 2961/2-1) and Ulrike Zetsche (DFG-ZE 1029/2-1).

\section{Supplemental Material}


RUNNING HEAD: AFFECT REGULATION IN BPD AND MDD

\section{References}

Abela, J. R. Z., Payne, A. V. L., \& Moussaly, N. (2003). Cognitive Vulnerability to Depression in Individuals With Borderline Personality Disorder. Journal of Personality Disorders, 17(4), 319-329. https://doi.org/10.1521/pedi.17.4.319.23968

Beblo, T., Fernando, S., Kamper, P., Griepenstroh, J., Aschenbrenner, S., Pastuszak, A., Schlosser, N., \& Driessen, M. (2013). Increased attempts to suppress negative and positive emotions in borderline personality disorder. Psychiatry Research, 210(2), $505-509$.

Beblo, T., Pastuszak, A., Griepenstroh, J., Fernando, S., Driessen, M., Schütz, A., Rentzsch, K., \& Schlosser, N. (2010). Self-reported emotional dysregulation but no impairment of emotional intelligence in borderline personality disorder: an explorative study. The Journal of Nervous and Mental Disease, 198(5), 385-388.

Ben-Zeev, D., \& Young, M. A. (2010). Accuracy of hospitalized depressed patients' and healthy controls' retrospective symptom reports: an experience sampling study. The Journal of Nervous and Mental Disease, 198(4), 280-285.

Bürkner, P.-C. (2017). brms: An R Package for Bayesian Multilevel Models Using Stan. Journal of Statistical Software, 80(1), 1-28. https://doi.org/10.18637/jss.v080.i01

Bürkner, P.-C. (2018). Advanced Bayesian Multilevel Modeling with the R Package brms. The R Journal, 10(1), 395-411.

Carpenter, B., Gelman, A., Hoffman, M. D., Lee, D., Goodrich, B., Betancourt, M., Brubaker, M., Guo, J., Li, P., \& Riddell, A. (2017). Stan: A probabilistic programming language. Journal of Statistical Software, 76(1).

Chapman, A. L., Rosenthal, M. Z., Dixon-Gordon, K. L., Turner, B. J., \& Kuppens, P. (2016). Borderline Personality Disorder and the Effects of Instructed Emotional Avoidance or Acceptance in Daily Life. Journal of Personality Disorders, 31(4), 483-502. https://doi.org/10.1521/pedi_2016_30_264 
RUNNING HEAD: AFFECT REGULATION IN BPD AND MDD

Daros, A. R., \& Williams, G. E. (2019). A meta-analysis and systematic review of emotionregulation strategies in borderline personality disorder. Harvard Review of Psychiatry, 27(4), 217-232.

Dell’Osso, L., Cremone, I. M., Carpita, B., Dell’Oste, V., Muti, D., Massimetti, G., Barlati, S., Vita, A., Fagiolini, A., Carmassi, C., \& Gesi, C. (2019). Rumination, posttraumatic stress disorder, and mood symptoms in borderline personality disorder.

Neuropsychiatric Disease and Treatment, 15, 1231-1238.

https://doi.org/10.2147/NDT.S198616

Dixon-Gordon, K. L., Aldao, A., \& Reyes, A. D. L. (2015). Repertoires of emotion regulation: A person-centered approach to assessing emotion regulation strategies and links to psychopathology. Cognition and Emotion, 29(7), 1314-1325. https://doi.org/10.1080/02699931.2014.983046

Ebner-Priemer, U. W., Kuo, J., Kleindienst, N., Welch, S. S., Reisch, T., Reinhard, I., Lieb, K., Linehan, M. M., \& Bohus, M. (2007). State affective instability in borderline personality disorder assessed by ambulatory monitoring. Psychological Medicine, 37(7), 961-970. https://doi.org/10.1017/S0033291706009706

Ebner-Priemer, U. W., Kuo, J., Welch, S. S., Thielgen, T., Witte, S., Bohus, M., \& Linehan, M. M. (2006). A valence-dependent group-specific recall bias of retrospective selfreports: A study of borderline personality disorder in everyday life. The Journal of Nervous and Mental Disease, 194(10), 774-779.

Evans, D., Howard, M., Dudas, R., Denman, C., \& Dunn, B. D. (2013). Emotion Regulation in Borderline Personality Disorder: Examining the Consequences of Spontaneous and Instructed Use of Emotion Suppression and Emotion Acceptance when Viewing Negative Films. Journal of Experimental Psychopathology, 4(5), 546-565. https://doi.org/10.5127/jep.029512 
RUNNING HEAD: AFFECT REGULATION IN BPD AND MDD

Fernando, S. C., Beblo, T., Schlosser, N., Terfehr, K., Otte, C., Löwe, B., Wolf, O. T., Spitzer, C., Driessen, M., \& Wingenfeld, K. (2014). The Impact of Self-Reported Childhood Trauma on Emotion Regulation in Borderline Personality Disorder and Major Depression. Journal of Trauma \& Dissociation, 15(4), 384-401. https://doi.org/10.1080/15299732.2013.863262

Fydrich, T., Renneberg, B., Schmitz, B., \& Wittchen, H.-U. (1997). Strukturiertes Klinisches Interview für DSM-IV Achse II: Persönlichkeitsstörungen. Hogrefe.

Gross, J. J. (1998). The emerging field of emotion regulation: An integrative review. Review of General Psychology, 2(3), 271-299.

Heiy, J. E., \& Cheavens, J. S. (2014). Back to basics: a naturalistic assessment of the experience and regulation of emotion. Emotion, 14(5), 878.

Joormann, J., \& Stanton, C. H. (2016). Examining emotion regulation in depression: A review and future directions. Behaviour Research and Therapy, 86, 35-49. https://doi.org/10.1016/j.brat.2016.07.007

Kircanski, K., Thompson, R. J., Sorenson, J. E., Sherdell, L., \& Gotlib, I. H. (2015). Rumination and Worry in Daily Life: Examining the Naturalistic Validity of Theoretical Constructs. Clinical Psychological Science, 3(6), 926-939. https://doi.org/10.1177/2167702614566603

Koole, S. L. (2009). The psychology of emotion regulation: An integrative review. Cognition \& Emotion, 23(1), 4-41. https://doi.org/10.1080/02699930802619031

Krause-Utz, A., Walther, J.-C., Lis, S., Schmahl, C., \& Bohus, M. (2019). Heart rate variability during a cognitive reappraisal task in female patients with borderline personality disorder: the role of comorbid posttraumatic stress disorder and dissociation. Psychological Medicine, 49(11), 1810-1821. 
RUNNING HEAD: AFFECT REGULATION IN BPD AND MDD

Lang, S., Kotchoubey, B., Frick, C., Spitzer, C., Grabe, H. J., \& Barnow, S. (2012). Cognitive reappraisal in trauma-exposed women with borderline personality disorder. NeuroImage, 59(2), 1727-1734. https://doi.org/10.1016/j.neuroimage.2011.08.061

Lincoln, T. M., Schulze, L., \& Renneberg, B. (2022). The role of emotion regulation in the characterization, development and treatment of psychopathology. Nature Reviews Psychology, 1(5), 272-286. https://doi.org/10.1038/s44159-022-00040-4

Linehan, M. M. (1993). Cognitive-behavioral treatment of borderline personality disorder. Diagnosis and treatment of mental disorders. Guilford Press.

Liu, D. Y., \& Thompson, R. J. (2017). Selection and implementation of emotion regulation strategies in major depressive disorder: An integrative review. Clinical Psychology Review, 57, 183-194. https://doi.org/10.1016/j.cpr.2017.07.004

Lobbestael, J., Leurgans, M., \& Arntz, A. (2011). Inter-rater reliability of the Structured Clinical Interview for DSM-IV Axis I Disorders (SCID I) and Axis II Disorders (SCID II). Clinical Psychology \& Psychotherapy, 18(1), 75-79. https://doi.org/10.1002/cpp.693

Nelson, J., Klumparendt, A., Doebler, P., \& Ehring, T. (2018). Everyday emotional dynamics in major depression. Emotion (Washington, DC).

Nolen-Hoeksema, S. (2000). The role of rumination in depressive disorders and mixed anxiety/depressive symptoms. Journal of Abnormal Psychology, 109(3), 504.

Nolen-Hoeksema, S., Wisco, B. E., \& Lyubomirsky, S. (2008). Rethinking rumination. Perspectives on Psychological Science, 3(5), 400-424.

Panaite, V., Koval, P., Dejonckheere, E., \& Kuppens, P. (2018). Emotion regulation and mood brightening in daily life vary with depressive symptom levels. Cognition and Emotion. psyh. https://doi.org/10.1080/02699931.2018.1543180

R Core Team. (2020). A language and environment for statistical computing. R Foundation for Statistical Computing. https://www.R-project.org/ 
RUNNING HEAD: AFFECT REGULATION IN BPD AND MDD

Ruscio, A. M., Gentes, E. L., Jones, J. D., Hallion, L. S., Coleman, E. S., \& Swendsen, J. (2015). Rumination predicts heightened responding to stressful life events in major depressive disorder and generalized anxiety disorder. Journal of Abnormal Psychology, 124(1), 17.

Santangelo, P., Reinhard, I., Mussgay, L., Steil, R., Sawitzki, G., Klein, C., Trull, T. J., Bohus, M., \& Ebner-Priemer, U. W. (2014). Specificity of affective instability in patients with borderline personality disorder compared to posttraumatic stress disorder, bulimia nervosa, and healthy controls. Journal of Abnormal Psychology, 123(1), 258-272. https://doi.org/10.1037/a0035619

Sauer, C., Sheppes, G., Lackner, H. K., Arens, E. A., Tarrasch, R., \& Barnow, S. (2016). Emotion regulation choice in female patients with borderline personality disorder: Findings from self-reports and experimental measures. Psychiatry Research, 242, 375-384. https://doi.org/10.1016/j.psychres.2016.04.113

Schulze, L., Bürkner, P.-C., Bohländer, J., \& Zetsche, U. (2018). Cognitive control and daily affect regulation in major depression and borderline personality disorder: protocol for an experimental ambulatory assessment study in Berlin, Germany. BMJ Open, 8(10), e022694. https://doi.org/10.1136/bmjopen-2018-022694

Selby, E. A., Anestis, M. D., Bender, T. W., \& Joiner Jr., T. E. (2009). An exploration of the emotional cascade model in borderline personality disorder. Journal of Abnormal Psychology, 118(2), 375-387. https://doi.org/10.1037/a0015711

Sheppes, G., Suri, G., \& Gross, J. J. (2015). Emotion Regulation and Psychopathology. Annual Review of Clinical Psychology, 11(1), 379-405. https://doi.org/10.1146/annurev-clinpsy-032814-112739

Skre, I., Onstad, S., Torgersen, S., \& Kringlen, E. (1991). High interrater reliability for the Structured Clinical Interview for DSM-III-R Axis I (SCID-I). Acta Psychiatrica Scandinavica, 84(2), 167-173. https://doi.org/10.1111/j.1600-0447.1991.tb03123.x 
RUNNING HEAD: AFFECT REGULATION IN BPD AND MDD

Sloan, E., Hall, K., Moulding, R., Bryce, S., Mildred, H., \& Staiger, P. K. (2017). Emotion regulation as a transdiagnostic treatment construct across anxiety, depression, substance, eating and borderline personality disorders: A systematic review. Clinical Psychology Review, 57, 141-163. https://doi.org/10.1016/j.cpr.2017.09.002

Steenkamp, M. M., Suvak, M. K., Dickstein, B. D., Shea, M. T., \& Litz, B. T. (2015). Emotional functioning in obsessive-compulsive personality disorder: Comparison to borderline personality disorder and healthy controls. Journal of Personality Disorders, 29(6), 794-808.

Svaldi, J., Dorn, C., Matthies, S., \& Philipsen, A. (2012). Effects of suppression and acceptance of sadness on the urge for non-suicidal self-injury and self-punishment. Psychiatry Research, 200(2), 404-416. https://doi.org/10.1016/j.psychres.2012.06.030

Svaldi, J., Griepenstroh, J., Tuschen-Caffier, B., \& Ehring, T. (2012). Emotion regulation deficits in eating disorders: A marker of eating pathology or general psychopathology? Psychiatry Research, 197(1), 103-111. https://doi.org/10.1016/j.psychres.2011.11.009

Vehtari, A., Gelman, A., Simpson, D., Carpenter, B., \& Bürkner, P.-C. (2021). RankNormalization, Folding, and Localization: An Improved R^ for Assessing Convergence of MCMC. Bayesian Analysis, 1(1), 1-28. https://doi.org/10.1214/20BA1221

Visted, E., Vøllestad, J., Nielsen, M. B., \& Schanche, E. (2018). Emotion Regulation in Current and Remitted Depression: A Systematic Review and Meta-Analysis. Frontiers in Psychology, 9, 756. https://doi.org/10.3389/fpsyg.2018.00756

Webb, T. L., Miles, E., \& Sheeran, P. (2012). Dealing with feeling: A meta-analysis of the effectiveness of strategies derived from the process model of emotion regulation. Psychological Bulletin, 138(4), 775-808. https://doi.org/10.1037/a0027600

Wittchen, H.-U., Wunderlich, U., Gruschwitz, S., \& Zaudig, M. (1997). SKID I. Strukturiertes Klinisches Interview für DSM-IV. Achse I: Psychische Störungen. Interviewheft und 
RUNNING HEAD: AFFECT REGULATION IN BPD AND MDD

Beurteilungsheft. Eine deutschsprachige, erweiterte Bearb. d. amerikanischen Originalversion des SKID I. Hogrefe.

Zaki, L. F., Coifman, K. G., Rafaeli, E., Berenson, K. R., \& Downey, G. (2013). Emotion differentiation as a protective factor against nonsuicidal self-injury in borderline personality disorder. Behavior Therapy, 44(3), 529-540.

Zetsche, U., Bürkner, P.-C., \& Renneberg, B. (2019). Future expectations in clinical depression: Biased or realistic? Journal of Abnormal Psychology, 128(7), 678-688. https://doi.org/10.1037/abn0000452 